\title{
El manejo de la atresia intestinal tipo IIIb sigue siendo un reto
}

\author{
Martín Gómez ${ }^{1}$, Tatiana Carolina Beltrán²
}

\section{Resumen}

La atresia intestinal constituye una de las causas más frecuentes de obstrucción intestinal en el recién nacido y es causada por catástrofes vasculares mesentéricas tardías durante el desarrollo embrionario; sin embargo, puede tener otras causas. La atresia de tipo IIIb es rara y su manejo es un reto para todo el equipo médico.

Este tipo de atresia sigue siendo de difícil manejo y requiere un equipo completo interdisciplinario para obtener un buen resultado: en primer lugar, lograr la supervivencia y, posteriormente, lograr un crecimiento y un desarrollo normales. La técnica quirúrgica incluye una anastomosis término-terminal; en complicaciones como las del caso presentado, se recurre a las plastias en YV, y a la colocación de férula en el intestino con sonda fenestrada para proteger la anastomosis, con el fin de mantener descomprimido el intestino y permeable distalmente.

Palabras clave: atresia intestinal, síndrome del intestino corto, obstrucción intestinal.

\section{Title}

The management of Type III intestinal atresia still remains being a challenge

\begin{abstract}
Intestinal atresia constitutes one of the most frequent causes of intestinal obstruction in the newborn and it is caused by delayed mesenteric vascular catastrophes during embryonic development; nevertheless, it may have other causes. Atresia type IIIb is rare and its management is a challenge for all of the members of the medical team.
\end{abstract}

1 Cirujano pediátrico, Hospital Universitario San Ignacio, Hospital Militar Central, Bogotá, D.C., Colombia.

2 Médica y cirujana, Pontificia Universidad Javeriana, Hospital Universitario San Ignacio, Bogotá, D.C., Colombia. 
This type of atresia continues being of difficult management, and needs a complete multidisciplinary equipment in order to attain good results: firstly, to ensure the survival of the child and subsequently; to achieve normal growth and development. The surgical technique includes endto- end anastomoses and, in complications like those presented in this case, we place YV shaped plasty and making an intestinal splint with a fenestrated probe in order to protect the anastomosis, maintaining bowel decompressed and distally-permeable.

Key words: intestinal atresia, short bowel syndrome, intestinal obstruction.

\section{Introducción}

La incidencia mundial de atresia intestinal tipo IIIb es de 2,25 por cada 10.000 nacidos vivos y, en Latinoamérica, es de 1,3 por 10.000 nacidos vivos. La frecuencia, según su localización, es de $50 \%$ en duodeno, $36 \%$ en yeyuno e íleon, $7 \%$ en colon y sólo 5\% de los casos corresponde a atresia múltiple. Es más frecuente en los prematuros $(35,4 \%)$ y en los de peso bajo al nacer (52\%)[1].

La atresia intestinal de tipo IIIb no es muy frecuente y puede ser de difícil diagnóstico; el manejo quirúrgico conjuga la habilidad y el cuidado de los tejidos y sus anastomosis.

En la primera intervención que se le practicó al paciente, se hizo una plastia y anastomosis término-terminal que, posteriormente presentó estenosis; en la segunda, se hizo una plastia en YV, ferulada con una sonda fenestrada y se obtuvo un buen resultado. Posteriormente, se inició la alimentación, primero parenteral total y luego entérica, con leche materna y aminoácidos libres.

\section{Presentación del caso}

Se trata de una paciente de sexo femenino, hija primogénita a término de una madre de 16 años y un padre de 23 años, sanos. Asistió a seis controles prenatales de alto riesgo por diagnóstico ecográfico de atresia intestinal y por ser producto de madre adolescente, quien presentó infección de vías urinarias en el primer trimestre que fue tratada con ampicilina y con urocultivo posterior negativo. Se hizo tamización para TORCH, VIH y VDRL, la cual fue negativa.

Nació por parto eutócico, con un puntaje de Apgar de 9/10 al minuto y 10/10a los 5 minutos, peso de 2.500 $\mathrm{g}$, talla de $46 \mathrm{~cm}$, perímetro abdominal de $32 \mathrm{~cm}$ y una escala de Ballard para 37 semanas. No se observó diuresis ni deposición de meconio. Al revisar la permeabilidad del esófago, hubo salida de un líquido amarilloverdoso por la sonda. Se encontró distensión abdominal, abdomen blando y ausencia de ruidos intestinales. El resto del examen clínico fue normal.

Se trasladó a la unidad de cuidados intensivos neonatales. Se mantuvo sin vía oral y se iniciaron líquidos 
intravenosos y un flujo metabólico de 5 a $8 \mathrm{mg} / \mathrm{kg}$ por minuto.

Se tomaron muestras para exámenes de laboratorio con los siguientes resultados: hematocrito de $39,8 \%$, hemoglobina de $13,7 \mathrm{mg} / \mathrm{dl}$ y el recuento de plaquetas fue normal; la hemoclasificación correspondió a A positivo; la tirotropina (TSH) neonatal y los tiempos de coagulación fueron normales.

La radiografía de abdomen sugería obstrucción intestinal mecánica alta (figura 1). Con la sonda orogástrica se obtuvo líquido de drenaje verde que requirió reposición 1:1; no se observó meconio. El concepto de cirugía pediátrica fue que se trataba de un cuadro de atresia intestinal, por lo cual se propuso practicar cirugía.

A las 37 horas de vida, se practicó la exploración quirúrgica y se encontró atresia yeyuno-íleal en cáscara de manzana (figura 2) y atresia de tipo 1 distal. Después de la liberación de bridas, se realizó plastia en YV de la atresia de tipo 1, y anastomosis con plastia del segmento proximal en la de tipo IIIb, con cierre del defecto del mesenterio y posterior colocación de sonda fenestrada en el intestino proximal. El estudio anatomopatológico del bloque de intestino informó:
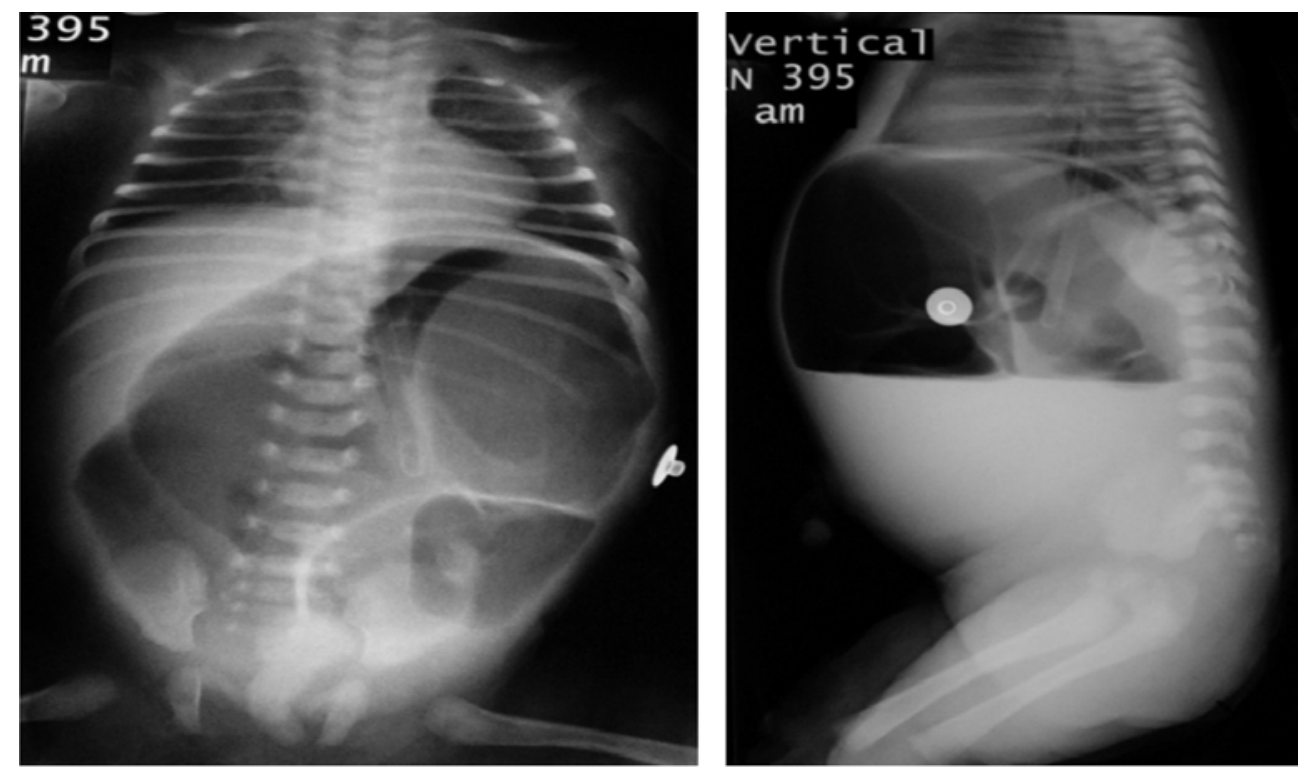

Figura 1. Radiografía de abdomen antero-posterior y lateral en vertical En las que se observa importante distensión de asas intestinales que parecen corresponder al intestino delgado, con ausencia de gas distal. 
"extremo proximal de características normales y extremo distal con extensa necrosis de coagulación".

En el periodo posoperatorio, hubo drenaje de escaso líquido serohemático por la sonda yeyunal y, posteriormente, hubo salida de un líquido amarillo-verdoso; se continuó la reposición 1:1. En los exámenes de laboratorio se encontraron hemograma, azoados, glucometrías, sodio, potasio, cloro, magnesio y transaminasas dentro de límites normales. Los resultados de los gases arteriales fueron: $\mathrm{pH}$ de $7,287, \mathrm{PCO}_{2}$ de $38,8 \mathrm{~mm} \mathrm{Hg}, \mathrm{PO}_{2}$ de $46 \mathrm{~mm} \mathrm{Hg}$ y $\mathrm{HCO}_{3}$ de $18,5 \mathrm{mmol} /$
L. La calcemia fue de $7,8 \mathrm{mg} / \mathrm{dl}$, la bilirrubina total de $4,5 \mathrm{mg} / \mathrm{dl}$, la directa de $0,46 \mathrm{mg} / \mathrm{dl}$ y la indirecta de 4,04 $\mathrm{mg} / \mathrm{d}$, que se encontraban en zona I para la edad. Se inició nutrición parenteral.

La evolución fue favorable. Se inició el cierre intermitente de la sonda orogástrica. Al octavo día del posoperatorio se realizó estímulo anal y presentó tres deposiciones de meconio con sangre negra. El drenaje por la sonda disminuyó progresivamente, apareció peristalsis intestinal y hubo adecuada tolerancia de la nutrición parenteral total.

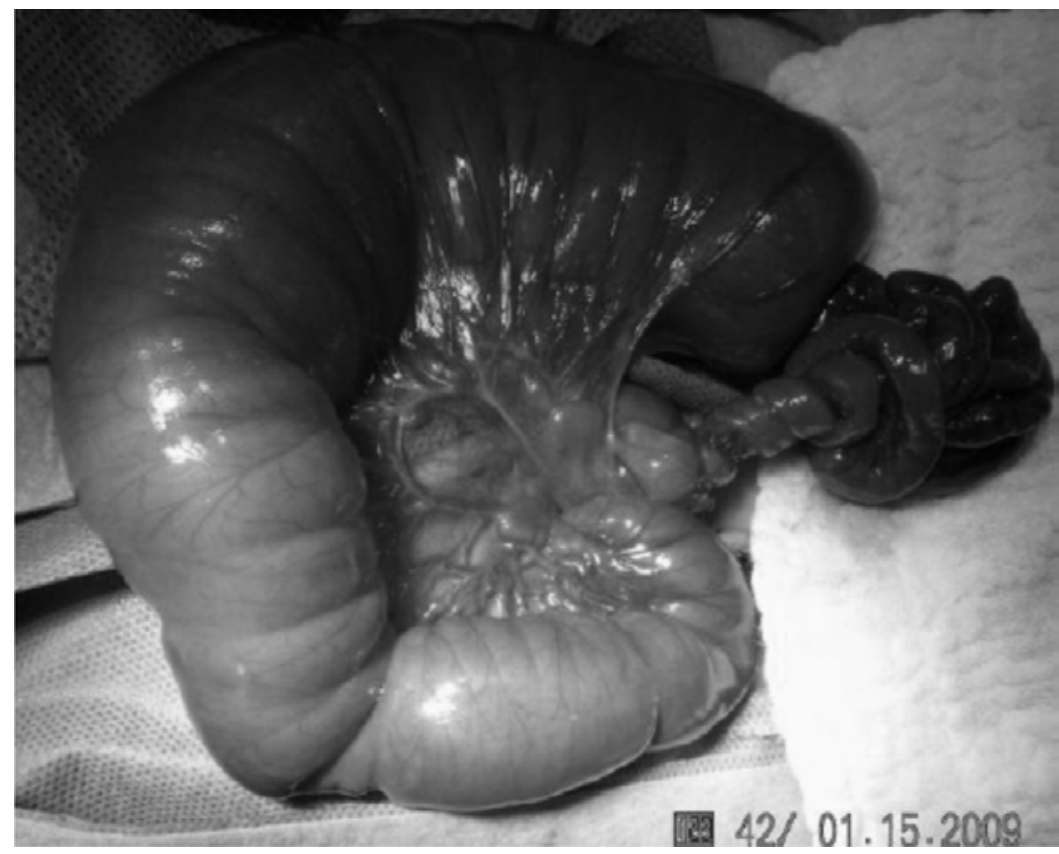

Figura 2. Dilatación del segmento proximal y un segmento estrecho enrollado sobre su propia irrigación. 
En el ecocardiograma se informó una comunicación interauricular de tipo ostium secundum de $5 \mathrm{~mm}$ de diámetro, sin repercusión hemodinámica, y buena función biventricular sin signos de hipertensión pulmonar.

En el décimo día posoperatorio se retiró la sonda; en el duodécimo día presentó picos febriles, hipoactividad, necesidad de oxígeno suplementario e íleo persistente. Requirió transfusión de glóbulos rojos.

En el decimotercer día los hemocultivos informaron cocos Gram positivos. Se inició cubrimiento antibiótico con piperacilina tazobactam, que posteriormente se cambió a vancomicina. El urocultivo y el cultivo de la punta del catéter fueron negativos. Continuó con distensión abdominal.

En el decimocuarto día se practicó estudio radiológico de tránsito intestinal con doble contraste, que evidenció obstrucción completa del yeyuno proximal (figura 3 ). Se colocó una sonda orogástrica que drenó abundante de líquido verde oscuro; no presentaba deposición y no había ruidos intestinales. Se demostró la presencia

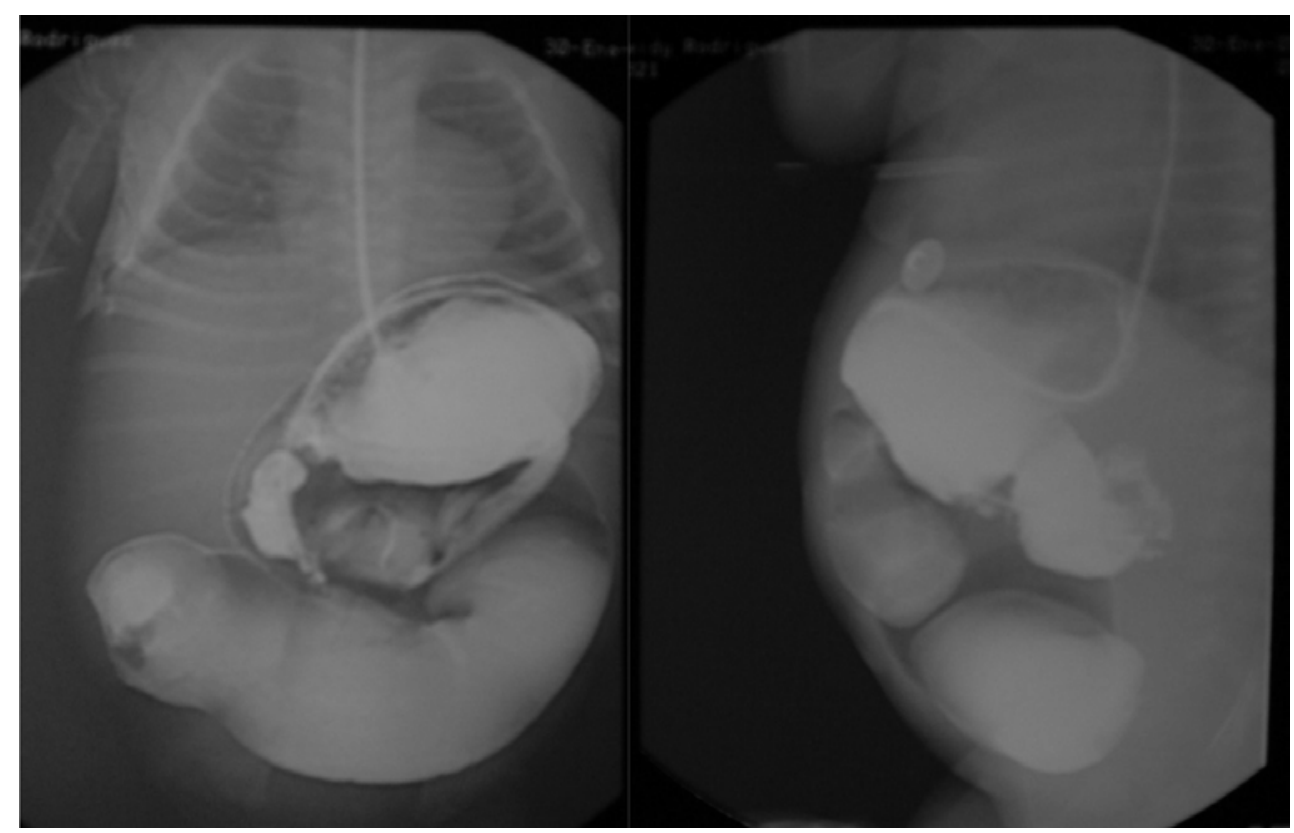

Figura 3. Tránsito intestinal con medio de contraste hidrosoluble. Se observa la cámara gástrica dilatada, con adecuado paso del medio de contraste hacia el duodeno, el cual es de curso y calibre normales. Hay dilatación de las asas intestinales del yeyuno proximal y obliteración abrupta, sin paso del medio en sentido distal. Los controles radiográficos cada 30 minutos durante dos horas no mostraron progresión del medio. Había obstrucción completa de las asas intestinales del yeyuno proximal. 
de Staphylococcus epidermidis sensible a la vancomicina.

Con el diagnóstico de obstrucción completa, a los 21 días de vida requirió una nueva cirugía, en la que se encontró obstrucción intestinal por bridas y estenosis de la anastomosis yeyuno-íleal. Se practicó liberación de las adherencias y una plastia en YV, y se pasó una sonda fenestrada hasta el ciego (figura 4). Recibió una nueva transfusión sanguínea.

La evolución posoperatoria fue buena inicialmente, durante el posoperatorio, presentó episodios eméticos, y el drenaje por la sonda y la disten- sión abdominal disminuyeron en forma progresiva. Recibió antibióticos durante 14 días. Estuvo en ayuno durante siete días y luego continuó con nutrición parenteral total, la cual toleró adecuadamente. Se le hizo estímulo anal y se obtuvo meconio. A los 28 días de vida, se inició la vía oral y recibió productos procinéticos, con buena tolerancia. El perfil metabólico mostró un patrón colestásico secundario a nutrición parenteral total prolongada.

A los 36 días de vida, se practicó un estudio de colon por enema que mostró cambios por desuso (figura 5). Se continuó con estímulo rectal y la-

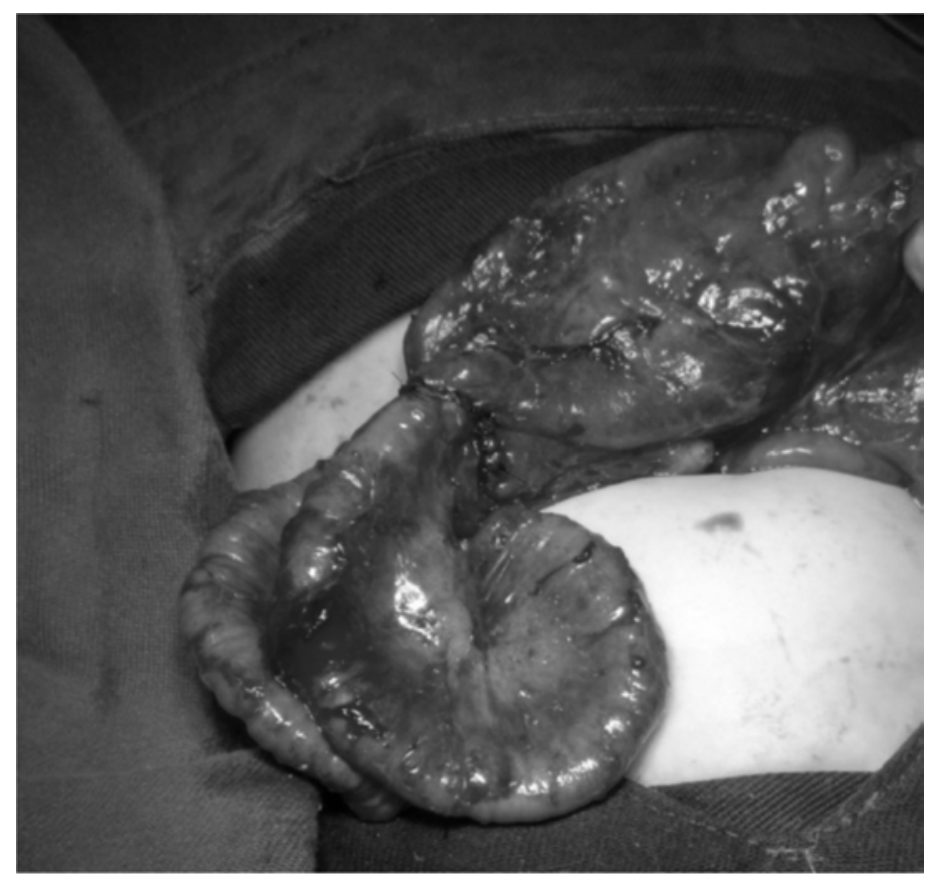

Figura 4. Anastomosis término-terminal yeyuno-ileal, con sonda fenestrada. 
vado rectal con solución salina, y se obtuvieron deposiciones. El aporte entérico máximo tolerado fue de 10,6 $\mathrm{ml}$ por hora en infusión continua por sonda orogástrica. A los 45 días, se inició alimentación con neocate según la tolerancia y tres días después se agregó piperacilina tazobactam para cubrir Gram negativos, por sospecha de traslocación bacteriana intestinal; no obstante, dada la buena evolución clínica y los resultados de los cultivos, se suspendieron y se continuó únicamente con vancomicina.

El aporte entérico se fue aumentando en forma progresiva, con periodos de distensión y vómito. El manejo posoperatorio continuó con soporte nutricional y aumento progresivo de la vía entérica y alimentación con seno materno; presentó periodos de intolerancia que obligaron a disminuir el aporte entérico, hasta llegar a tolerar su aporte necesario y lograr destete de la nutrición parenteral total. La estancia hospitalaria fue de 59 días. Al egreso su estado era satisfactorio.

\section{Discusión}

El diagnóstico oportuno, la mayoría de las veces prenatal como en este caso, lleva a mejorar las posibilidades de supervivencia de los pacientes con enfermedad congénita, cuando el control prenatal y el parto se llevan a cabo en centros especializados con manejo de alto riesgo. Las unidades de cuidado intensivo neonatal ofrecen garan-

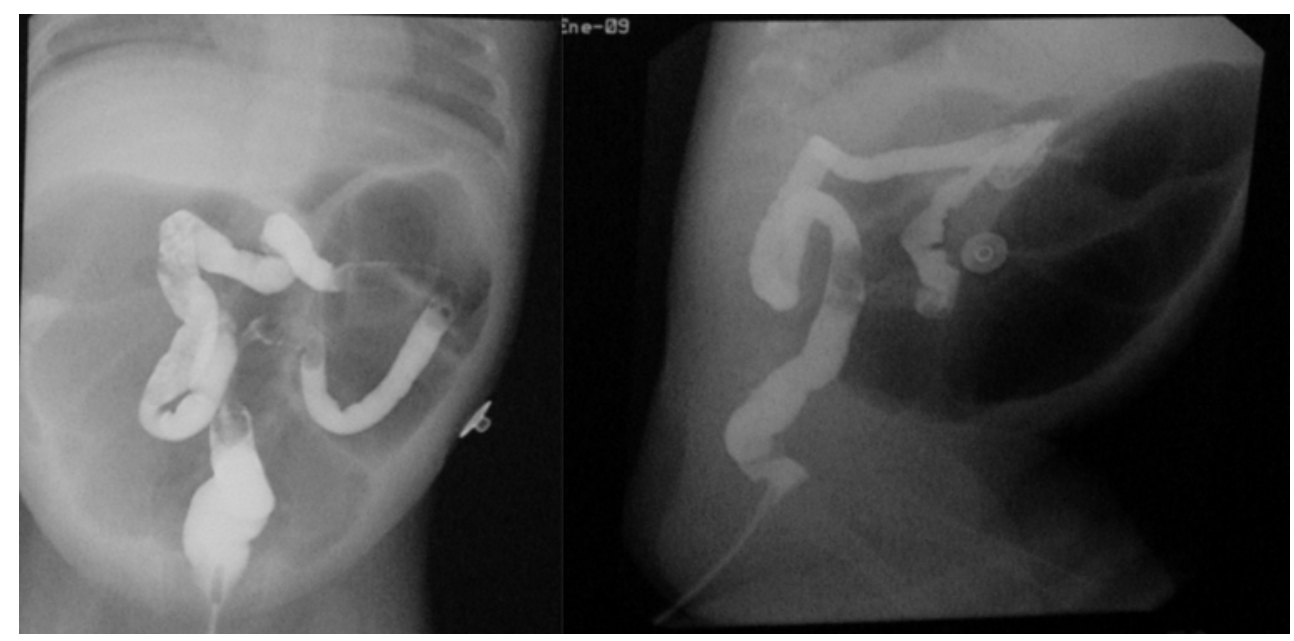

Figura 5. Radiografía de colon por enema con doble contraste, con medio de contraste yodado hidrosoluble. Se observa importante disminución del calibre de los diferentes segmentos del colon, sin zonas de estenosis o trayectos fistulosos. El calibre desde el ciego hasta el colon sigmoide no es mayor de $8 \mathrm{~mm}$ y el de la ampolla rectal alcanza $13 \mathrm{~mm}$. Se hizo diagnóstico de microcolon por desuso. 
tía para el manejo antes de la cirugía y después de ella; se pueden hacer los estudios complementarios necesarios y así llevar estos pacientes en las mejores condiciones al quirófano, donde se ponen a prueba las habilidades del cirujano al realizar las anastomosis conservando todo el intestino posible.

Esta variedad de atresia cursa con el síndrome de intestino corto, por lo cual las ostomías están contraindicadas. En casos como éste en que se esperan dificultades, actualmente se están usando rutinariamente férulas en todas las anastomosis, con sonda fenestrada. Se debe hacer el diagnóstico diferencial con la enfermedad de Hirschsprung y descartar otras atresias; en este caso existía una tipo 1 distal, que también fue corregida con una plastia en $\mathrm{YV}$, con resección de la membrana.

El control posoperatorio debe hacerse en la unidad de cuidados intensivos y hay que estar pendientes de las posibles complicaciones para atenderlas en forma precoz y adecuada. La suma de todos estos esfuerzos lleva a mejorar la supervivencia.

\section{Conclusión}

La atresia intestinal de tipo IIIb continúa siendo un reto para todo el equipo médico que maneja patología quirúrgica del recién nacido. Las faci- lidades del diagnóstico prenatal, la mejoría en la conducción del embarazo y el parto, la existencia de unidades de cuidados intensivos neonatales y el apoyo interdisciplinario en el manejo integral, mejoran la supervivencia y la calidad de vida.

Se debe conservar la mayor cantidad de intestino y, especialmente, la válvula ileocecal; en el manejo de intestino corto durante el posoperatorio, ese detalle técnico juega un papel preponderante.

\section{Agradecimientos}

Al Departamento de Radiología, a la Unidad de Cuidados Intensivos Neonatales del Hospital Universitario San Ignacio y al Departamento de Pediatría del Hospital Universitario San Ignacio, y al doctor Luis Daniel Abril.

\section{Bibliografía}

1. Reynoso E, Camargo FD Gaona, Rangel MC, Guzmán AN. Atresia intestinal múltiple: Rev Med Hosp Gen Mex. 2008;71:94-8.

2. De Grazia E, Di Pace MR, Caruso AM, Catalano P, Cimador M. Different types of intestinal atresia in identical twins. J Pediatr Surg. 2008;43:2301-

3. Komuro H, Amagai T, Hori T, Hirai M, Matoba K, Watanabe M, et al. Placental vascular compromise in jejunoileal atresia. J Pediatr Surg. 2004;39:1701-5. 
4. Komuro H, Hori T, Amagai T, Hirai M, Yotsumoto K, Urita Y, et al. The etiologic role of intrauterine volvulus and intussusception in jejunoileal atresia. J Pediatr Surg. 2004;39:1812-4.

5. Federici S, Domenichelli V, Antonellini C, Dòmini R. Multiple intestinal atresia with apple peel syndrome: successful treatment by five end-to-end anastomoses, jejunostomy and transanastomotic silicone stent. J Pediatr Surg. 2003;38:1250-2.

6. Festen S, Brevoord JC, Goldhoorn GA, Festen C, Hazebroek FW, van Heurn LW, et al. Excellent long-term outcome for survivors of apple peel atresia. $J$ Pediatr Surg. 2002;37:61-5.

7. Imaizumi K, Kimura J, Masuno M, Kuroki Y, Nishi T. Apple-peel intestinal atresia associated with balanced reciprocal translocation $\mathrm{t}(2 ; 3)(\mathrm{q} 31.3$; p24.2) mat. Am J Med Genet. 1999; 87:434-5.

8. Tam PK, Nicholls G. Implications of antenatal diagnosis of small-intestinal atresia in the 1990s. Pediatr Surg Int. 1999;15:486-7.

9. Weber DM, Freeman NV. Duodeno-jejunal atresia with apple peel configuration of the ileum and absent superior mesenteric artery: observations on pathogenesis. J Pediatr Surg. 1999; 34:1427-9.

10. Chaet MS, Warner BW, Sheldon CA. Management of multiple jejuno-ileal atresias with an intraluminal SILASTIC stent. J Pediatr Surg. 1994;29:1604-6.

11. Lamireau $\mathrm{T}$, Janoyer $\mathrm{M}$, Llanas $\mathrm{B}$, Vergnes P, Galperine I, Demarquez JL, et al. Apple-peel jejunal atresia. Nutritional management. Ann Pediatr (Paris). 1993;40:368-70.

12. Weissman A, Goldstein I. Prenatal sonographic diagnosis and clinical management of small bowel obstruction. Am J Perinatol. 1993;10:215-6.

13. Touloukian RJ. Diagnosis and treatment of jejujoileal atresia. World $J$ Surg. 1993;17:310-7.

14. Turnock RR, Brereton RJ, Spitz L, Kiely EM. Primary anastomosis in apple-peel bowel syndrome. J Pediatr Surg. 1991;26:718-20.

15. Grosfeld JL, Ballantine T, Shoemaker R. Intestinal operative management of atresia and stenosis based on pathologic findings. J Pediatr Surg. 1979; 14:368-74. 